\title{
Cambios en la personalidad y depresión en adultos mayores con enfermedad de Alzheimer
}

\author{
Changes in personality and depression in older adults with Alzheimer's disease
}

\author{
Charles Y. Da Silva Rodrigues ${ }^{1}$ \\ María Teresa Hernández Ramos ${ }^{2}$ \\ Paula A. Carvalho Figueiredo ${ }^{3}$ \\ Estela Romero Lara ${ }^{4}$ \\ ${ }^{1}$ Ciencias de la Salud - Psicología, Universidad de Guanajuato. México \\ ${ }^{2}$ Departamento de Enfermería y Obstetricia, Universidad de Guanajuato. México \\ ${ }^{3}$ Departamento de Ciencias Sociales y Gestión, Universidade Aberta. Portugal \\ ${ }^{4}$ Instituto Nacional de Personas Adultas Mayores de Guanajuato. México
}

\begin{abstract}
Resumen: La literatura refiere que el cambio de personalidad se inicia en la fase premórbida del trastorno neurocognitivo leve (TNC) y que la depresión puede incrementar esos cambios, por otro lado, la depresión es tratable y como tal su influencia en la personalidad es reversible. En este sentido urge entender cuál es la influencia efectiva de la depresión en el TNC, en pacientes con Alzheimer. Objetivo: Explorar nivel de depresión y cambios en la personalidad en pacientes adultos mayores con TNC debido a Enfermedad de Alzheimer. Metodología: Participaron 437 adultos mayores, evaluados a través de la aplicación del Inventario de Personalidad NEO-FFI; Examen del Estado Mental (MME), y el Inventario de Depresión de Beck (BDI- II). Resultados: Se verificó un incremento de cambios comportamentales por influencia del rasgo de responsabilidad, asociado al aumento de la sintomatología cognitiva, ambos por influencia de la depresión.
\end{abstract}

Palabras clave: deterioro cognitivo leve, trastorno neurocognitivo, personalidad, cognición, adulto mayor, depresión, enfermedad de Alzheimer

\begin{abstract}
The literature refers that personality change begins in the premorbid phase of mild neurocognitive disorder (TNC) and that depression can increase these changes, on the other hand, depression is treatable and as such its influence on personality is reversible. In this sense it is urgent to understand what the effective influence of depression in the TNC is, in patients with Alzheimer's. Objective: Explore level of depression and changes in personality in elderly with TNC due to Alzheimer's disease. Methodology: A total of 437 older adults participated. Participants were evaluated through the application of a Personality Inventory NEO-FFI; Mini-Mental State Examination (MMSE), and the Beck Depression Inventory (BDI-II). Results: There was an increase in behavioural changes due to the influence of the responsibility trait, associated with the increase in cognitive symptoms, both due to the influence of depression.
\end{abstract}

Keywords: mild cognitive impairment, neurocognitive disorder, personality, cognition, elderly, depression, Alzheimer's disease

Cómo citar este artículo:

Da Silva Rodrigues, Ch. Y., Hernández Ramos, M. T., Carvalho Figueiredo, P. A., \& Romero Lara, E. (2018). Cambios en la personalidad y depresión en adultos mayores con enfermedad de Alzheimer. Ciencias Psicológicas, 12(2), 231-237. doi: https://doi.org/10.22235/cp.v12i2.1687.

Correspondencia: Charles Y. Da Silva Rodrigues Universidad de Guanajuato, División de Ciencias de la Salud, Psicología, campus León; e-mail: charles.rodrigues@ugto.mx. María Teresa Hernández Ramos; e-mail: tita@ugto.mx. Paula A. Carvalho Figueiredo; e-mail:paulafigeuiredo@campus.ul.pt.Estela RomeroLara; e-mail:inapamgto@yahoo.com.mx 
Los trabajos previos en esta línea de investigación han demostrado que existen cambios en los rasgos de personalidad del adulto mayor con trastorno neurocognitivo (TNC) leve, aunque sea difícil de sistematizar un patrón de cambios (Riddle, Potter, McQuoid, Steffens, Beyer, \& Taylor, 2017; Rodrigues, Castro, \& Figueiredo, 2014). La personalidad tiene dos factores intrínsecos importantes que se basan en su propia constitución: temperamento y carácter. El temperamento está asociado a la dotación genética, en este sentido influye y se deja influir por las vivencias de cada individuo. En cambio, el carácter se relaciona con una combinación de sentimientos y comportamientos, considerarse como un aspecto importante, cuya naturaleza es individual, cultural y social (Cloninger, Przybeck, Svrakic, \& Wetzel, 1994; Da Silva, 2017).

La literatura refiere que el cambio se inicia en la fase premórbida del TNC y que está relacionado con una disminución de la responsabilidad, considerando las 5 dimensiones de la teoría del Big Five Factor (Costa, Terracciano, \& McCrae, 2001; Costa \& Widiger, 2002). Los rasgos de personalidad parecen ajustarse a un patrón de disminución de la responsabilidad, en fase premórbida, es decir, de antes del diagnóstico, hacia una disminución progresiva de la amabilidad y del estado cognitivo. Al parecer, los cambios cognitivos están asociados a la misma responsabilidad por alteraciones al nivel de la orientación espacial y capacidades visoconstructivas, y a la amabilidad por alteraciones de memoria inmediata y atención (Da Silva, Carvalho, González, \& Quino, 2018; Pocnet, Rossier, Antonietti, \& Gunten, 2013; Rodrigues, Castro, \& Gruart, 2014b; Vidovich \& Almeida, 2011).

Otras investigaciones se han orientado al TNC con deterioro cognitivo moderado y se ha verificado, que después del deterioro cognitivo leve (DCL), se incrementan los cambios en los rasgos de personalidad, con una disminución aún más acentuada de la responsabilidad, y cambios al nivel del neuroticismo y de la apertura (Da Silva, Carvalho, Quino, \& Gruart, 2017). Dichas investigaciones relacionan el empeoramiento del patrón conductual, con los cambios de los mecanismos de memoria y atención. Considerando, que esta relación pudiera ser de base para en entendimiento de los cambios neuropsiquiátricos, que a su vez se relación con los cambios conductuales asociados a la degeneración cerebral (Pérez-Fuentes, Gázquez, \& Morelo, 2012; Ramakers \& Verhey, 2017; Rodrigues, Castro, \& Cruz Roja Española, 2014).
En investigaciones previas pudimos verificar que la relación entre rasgos de personalidad y deterioro cognitivo se basan en una asociación de memoria inmediata en más de $6 \%$ del neuroticismo y más del $3 \%$ de la amabilidad; mientras la atención se asocia en más de un 3\% igualmente, con el neuroticismo (Da Silva, Ramos, \& Carvalho, 2018; Rodrigues, 2015). Aunque la relación no es clara cuanto a su causa efecto, considerando que también podría justificar los cambios de neuroticismo a las alteraciones cognitivas de la memoria y atención. Finalmente, se relacionan ambos incrementos de deterioro: rasgos de personalidad y alteraciones al nivel de los mecanismos cognitivos (Qazi, Gutzmann, \& Gul, 2017).

El análisis de los hallazgos de estas investigaciones previas a través de los conceptos teóricos del Big Five Factor, representan cambios conductuales, en la fase premórbida del adulto mayor, con una tendencia a dejar de planificar el futuro, a la perdida de interés por las cosas novedosas e inclusivamente, por aquellos asuntos que eran sus predilectos. Mientras que, en el DCL, los cambios premórbidos se incrementan y surge una tendencia para experimentar angustia y ansiedad, junto con dificultades para manejar el estrés y el control de impulsos. A estos cambios de personalidad se añaden las alteraciones de memoria y atención, y de otros mecanismos cognitivos en caso de depresión asociada (Da Silva \& Carvalho, 2017a; Ramakers \& Verhey, 2017; Rodrigues, Castro, \& Gruart, 2014a).

Alteraciones en los mecanismos cognitivos de orientación; atención y cálculo; lenguaje; memoria; y recuerdo diferido y capacidad viso-constructiva de los adultos mayores con Alzheimer en fase inicial, pueden presentar mayor intensidad sintomatológica del cuadro neurodegenerativo cuando esté asociado a la depresión (Da Silva \& Carvalho, 2017b). La depresión, en general, y bien como se considera en esta investigación, se instaura en el mayor cuando este empieza a ganar consciencia y a tener percepción de la enfermedad de Alzheimer y sus consecuencias. Así, investigaciones previas refieren que la depresión tanto de tipo leve como de tipo moderada generan alteraciones al nivel de los procesos cognitivos (Qazi, Gutzmann, \& Gul, 2017).

En este sentido parece fundamental conocer la etiología del estado depresivo para que la evaluación pueda ser precisa y así, proporcionar al adulto mayor o a su cuidador un diagnóstico diferencial. Un buen diagnóstico posibilita al profesional de salud una mejor adecuación del programa de es- 
timulación cognitiva, para que sea coherente con el cuadro neurodegenerativo, sin considerar la intensificación de los síntomas provocados por la depresión, y considerando que la depresión este siendo tratada. La depresión, se puede tratar y como tal, hacer reversible el incremento de las alteraciones cognitivas del adulto mayor y consecuentemente de los cambios conductuales (Da Silva, Ramos, \& Carvalho, 2018; Leyhe et al., 2017).

Es necesario añadir que cada adulto mayor tiene un envejecimiento particular que no puede ser generalizado o comparado al de otro mayor, situación que es aún más complicada cuando el envejecimiento está acompañado de la enfermedad de Alzheimer (Riddle et al., 2017). Lo más significativo es que al envejecer, la comorbilidad con mayor frecuencia es la depresión; aunque la depresión geriátrica puede estar asociada a otras comorbilidades, como las enfermedades que se van presentando como una cuestión propia de la edad. Finalmente, los criterios socioculturales, económicos y el apoyo de la familia serán fundamentales, principalmente al momento de la intervención (Da Silva, Ramos, \& Carvalho, 2018).

Así, este trabajo tuvo como objetivo explorar el nivel de depresión y los cambios en la personalidad en pacientes adultos mayores con trastorno neurocognitivo leve (TNC) debido a Enfermedad de Alzheimer.

\section{Método}

\section{Participantes}

La muestra fue de 437 mayores residentes en Guanajuato, México, con 239 mujeres (54.6\%) y 198 varones $(45.4 \%)$. La edad media de los participantes fue de 87 años y fueron divididos homogéneamente en dos grupos: un grupo experimental (GE) y un grupo de control (GC).

El GE de 210 adultos mayores con Alzheimer en fase inicial, DCL y con depresión escogidos aleatoriamente a través del Instituto Nacional de las Personas Adultas Mayores (INAPAM) del Estado de Guanajuato. El GE tenía una media de edad de 86 años que variaba entre los 74 y los 98 años, su división por género estaba representada por 120 mujeres $(57.1 \%)$ y 90 varones $(42.9 \%)$.

El GC fue constituido por 227 adultos mayores en fase de envejecimiento normal y sin depresión escogidos aleatoriamente a través del Instituto Nacional de las Personas Adultas Mayores (INAPAM) del Estado de Guanajuato. El GE tenía una media de edad de 88 años que variaba entre los 78 y los 97 años, su división por género estaba representada por 119 mujeres $(52.4 \%)$ y 108 varones $(47.6 \%)$.

Como criterios de inclusión, para el GE los participantes tuvieron que presentar un diagnóstico clínico de Alzheimer avalado por un neurólogo y un diagnóstico clínico de depresión avalado por un psiquiatra. El diagnóstico de depresión debería ser posterior al de Alzheimer, asegurando que la hipótesis de depresión se debe a la consciencia de la enfermedad. Para GC el único criterio de inclusión que tenían que presentar era que no padecieran de ningún tipo de enfermedad crónica grave, cognitiva o depresión, avalado clínicamente por el médico de familia. Todos los participantes tenían que tener 70 años o más para poder participar en la investigación.

\section{Instrumentos}

Para evaluar el nivel de depresión, se utilizó el Inventario de Depresión de Beck (BDI- II) creado en 1996. Es una de las pruebas más utilizadas a nivel mundial para población clínica y de investigación, considerando que aparece en muchos estudios y porque presenta buenos niveles de validez y confiabilidad, permitiendo la comparación con otros estudios. Consta de 21 reactivos, formulados con las descripciones verbales que generalmente la población clínica depresiva proporciona sobre su sintomatología. El inventario es de tipo Likert, de cuatro puntos, donde "0" es "no", y 3 es "sí, mucho". Su aplicación es corta, alrededor de 15 minutos, además puede ser auto aplicable (Beck, Steer, \& Brown, 1996; Beck, Ward, Mendelson, Mock, \& Erbaugh, 1961).

Mini Mental Examination (MME) de Folstein. El MME es un instrumento clínico de aplicación individual, con una duración aproximada de 15 minutos, que se utiliza para la valoración terapéutica de las funciones cognitivas de los pacientes, así como para verificar los niveles cognitivos y para el rastreo de las demencias. El MME está dividido en 5 categorías de evaluación: orientación; memoria; atención y cálculo; lenguaje; recuerdo diferido y capacidades viso-constructivas. La puntuación bruta podrá necesitar correcciones de acuerdo con la escolaridad y edad del usuario (Folstein, Folstein, \& McHugh, 1975).

Para realizar la evaluación de personalidad se utilizó el inventario de personalidad NEO-FFI, en su versión reducida de 60 ítems y cinco dimensiones o rasgos: neuroticismo, extroversión, apertura 
a la experiencia, amabilidad y responsabilidad. La validación del inventario presenta un nivel de homogeneidad de 0.22 a 0.45 y una consistencia interna de .70 a .71 , mientras que el coeficiente alfa de Cronbach $(\alpha)$ indica una fiabilidad de .73 para el rasgo de neuroticismo, de .78 para extroversión, de .79 para apertura a la experiencia, de .70 para amabilidad y de .74 para responsabilidad (Costa, Terracciano \& McCrae, 2001; Costa \& Widiger, 2002).

\section{Procedimiento}

Se explicitaron condiciones y objetivos del estudio a participantes y cuidadores, a la vez que se obtuvieron los Consentimientos y se tramitó Historial clínico, todo dentro del ámbito del INAPAM y según criterios APA y los de la Ley General de Salud de México. Se realizó la administración en 4 sesiones, la última destinada a informar sobre los resultados.

El análisis de los resultados se realizó en el programa Statistical Package for the Social Sciences (SPSS) en su versión número 22 para sistema Windows 7. Las pruebas estadísticas usadas fueron el coeficiente de correlación de Spearman con el 95\% de confianza, a su vez para contrastar las diferencias entre los distintos niveles de depresión y mecanismos cognitivos se realizó la prueba no paramétrica de Kruskal Wallis.

\section{Resultados}

En la tabla 1 se puede verificar el análisis de la relación entre ambos grupos al nivel de los rasgos de personalidad y los mecanismos cognitivos,

Tabla 1

Análisis de los rangos en la relación a la Personalidad y los mecanismos cognitivos

\begin{tabular}{lccc}
\hline & $\begin{array}{c}\text { Mecanismos } \\
\text { Cognitivos }\end{array}$ & $\mathrm{N}$ & $\begin{array}{c}\text { Rango } \\
\text { Promedio }\end{array}$ \\
\hline \multirow{3}{*}{ Neuroticismo nivel } & Normal alto & 289 & 249.70 \\
& Moderado & 144 & 259.58 \\
& Total & 437 & \\
& Normal alto & 79 & 254.64 \\
Extraversión nivel & Moderado & 24 & 243.31 \\
& Total & 437 & \\
& Normal alto & 79 & 254.09 \\
Apertura nivel & Moderado & 24 & 245.13 \\
& Total & 437 & \\
& Normal alto & 79 & 252.49 \\
Amabilidad nivel & Moderado & 24 & 250.40 \\
& Total & 437 & \\
& Normal alto & 79 & 248.99 \\
Responsabilidad nivel & Moderado & 24 & 261.92 \\
& Total & 437 & \\
\hline
\end{tabular}

comparados los grupos experimental y de control. En cada rasgo de personalidad del Big Five Factor se puede verificar el nivel del estado de los mecanismos cognitivos, considerando el nivel normal alto y moderado.

Se realizó una prueba para encontrar diferencias entre los entre los productos de los procesos cognitivos y los rasgos de personalidad según el NEO-FFI, filtrando aquellas personas que presentaban algún tipo de depresión medido por el BDI-II, encontrando diferencias estadísticamente significativas con la prueba de Kruskal Wallis entre el rasgo de responsabilidad $(p=.042)$ y un nivel moderado de deterioro (ver tabla 2).

Conforme referimos anteriormente existen fuertes relaciones entre cognición y depresión, bien como entre cognición y personalidad, en este sentido pareció fundamental indagar sobre la influencia que la depresión podrá tener sobre la cognición, considerando que el grupo experimental presenta DCL (ver tabla 3).

Los resultados obtenidos por medio del coeficiente de Spearman muestran que existe una correlación estadísticamente significativa entre el nivel de los procesos cognitivos y el nivel de depresión, a mayor deterioro cognitivo, mayores niveles de depresión $(r=.575, p=.000)$ cuando comparamos GE con GC.

En la tabla 4 se puede verificar el análisis entre los rangos de Memoria y Niveles de Depresión según BDI-II y entre GC y GE. Para cada Proceso cognitivo se realiza un estudio según nivel de depresión (sin depresión, depresión intermitente y depresión moderada).

Para contrastar diferencias entre los niveles de depresión medidos por el BDI-II; se realizó una prueba de Kruskal Wallis. Los resultados muestran la existencia de diferencias estadísticamente significativas entre las tareas de orientación $(p=.00)$, atención y cálculo $(p=.000)$, lenguaje $(p=.000)$, memoria $(p=.019)$, capacidades viso-constructivas $(p=.000)$ y recuerdo diferido $(p=.000)$.

En este sentido, puntuaron con menor desempeño aquellas personas que presentan una depresión moderada o intermitente en las tareas de orientación, atención y cálculo, lenguaje, capacidades viso-constructivas y memoria, exceptuando las tareas de capacidades viso-constructivas, donde las personas que no presentan depresión tienen menor puntaje que aquellas que presentan algún tipo de depresión medido por el BDI-II (ver tabla 5). 
Tabla 2

Estadísticos de prueba Kruskal Wallis

\begin{tabular}{lccccc}
\hline & $\begin{array}{c}\text { Neuroticismo } \\
\text { nivel }\end{array}$ & $\begin{array}{c}\text { Extraversión } \\
\text { nivel }\end{array}$ & $\begin{array}{c}\text { Apertura } \\
\text { nivel }\end{array}$ & $\begin{array}{c}\text { Amabilidad } \\
\text { nivel }\end{array}$ & $\begin{array}{c}\text { Responsabilidad } \\
\text { nivel }\end{array}$ \\
\hline Chi-cuadrado & 2.522 & 3.050 & 2.034 & .104 & 4.123 \\
gl & 1 & 1 & 1 & 1 & 1 \\
Sig. asintótica & .112 & .081 & .154 & .747 & .042 \\
\hline
\end{tabular}

a. Prueba de Kruskal Wallis

b. Variable de agrupación: estado mental

Tabla 3

Correlaciones mecanismos cognitivos con nivel de depresión

\begin{tabular}{|c|c|c|c|}
\hline & & & $\begin{array}{c}\text { Mecanismos } \\
\text { Cognitivos }\end{array}$ \\
\hline Rho de Spearman & $\begin{array}{l}\text { Nivel } \\
\text { depresión }\end{array}$ & $\begin{array}{l}\text { Coeficiente de correlación } \\
\text { Sig. (bilateral) } \\
\text { N }\end{array}$ & $\begin{array}{r}.559^{* *} \\
.000 \\
437 \\
\end{array}$ \\
\hline
\end{tabular}

Tabla 4

Análisis de los rangos en la relación de los mecanismos cognitivos y la depresión

\begin{tabular}{lccc}
\hline \multicolumn{1}{c}{ Cognición / Depresión } & Nivel depresión & $\mathrm{N}$ & Rango promedio \\
\hline \multirow{4}{*}{ Orientación } & Sin depresión & 273 & 119.88 \\
& Intermitente & 58 & 93.93 \\
& Moderado & 106 & 90.02 \\
& Total & 437 & \\
& Sin depresión & 273 & 238.79 \\
Atención y cálculo & Intermitente & 58 & 66.00 \\
& Moderado & 106 & 173.10 \\
& Total & 437 & \\
\hline \multirow{4}{*}{ Memoria } & Sin depresión & 273 & 216.26 \\
& Intermitente & 58 & 90.18 \\
& Moderado & 106 & 194.65 \\
& Total & 437 & \\
\hline \multirow{4}{*}{ Lenguaje } & Sin depresión & 273 & 237.89 \\
& Intermitente & 58 & 62.43 \\
& Moderado & 106 & 174.65 \\
& Total & 437 & \\
\hline \multirow{3}{*}{ Capacidades Viso-constructivas } & Sin depresión & 273 & 230.13 \\
& Intermitente & 58 & 78.32 \\
& Moderado & 106 & 180.92 \\
& Total & 437 & \\
\hline \multirow{2}{*}{ Recuerdo diferido } & Sin depresión & 273 & 85.76 \\
& Intermitente & 58 & 229.29 \\
& Moderado & 106 & 222.85 \\
\hline
\end{tabular}

Tabla 5

Estadísticos de prueba Kruskal Wallis

\begin{tabular}{lcccccc}
\hline & Orientación & $\begin{array}{c}\text { Atención y } \\
\text { Cálculo }\end{array}$ & Memoria & Lenguaje & $\begin{array}{c}\text { Capacidades Viso- } \\
\text { constructivas }\end{array}$ & $\begin{array}{c}\text { Recuerdo } \\
\text { diferido }\end{array}$ \\
\hline Chi-cuadrado & 20.745 & 66.602 & 7.972 & 62.356 & 40.779 & 22.369 \\
gl & 2 & 2 & 2 & 2 & 2 & 2 \\
Sig. asintótica & .000 & .000 & .019 & .000 & .000 & .000 \\
\hline
\end{tabular}

a. Prueba de Kruskal Wallis

b. Variable de agrupación: nivel depresión 


\section{Discusión}

En tanto la depresión puede ser tratada posibilita un reajuste en el deterioro cognitivo. Muchas veces en las evaluaciones de la personalidad este factor no se considera, siendo que el diagnóstico acaba por presentarse más grave de lo que realmente debería de ser. Investigaciones previas consideran la influencia de la depresión en los cambios cognitivos, sin dejar de considerar que una depresión posterior al diagnóstico de Alzheimer, estará fundamentalmente asociada a la percepción que el adulto mayor tiene de la enfermedad que padece (Da Silva 2017; Riddle et al.,2017; Rodrigues, Castro, \& Figueiredo, 2014).

Así, se pudo verificar un menor desempeño cognitivo en los adultos mayores que presentaron un nivel de depresión moderado o intermitente. El incremento de las limitaciones se verifica en orientación, atención y cálculo, lenguaje, capacidades viso-constructivas y memoria, siendo que únicamente los mecanismos cognitivos de capacidades viso-constructivas, donde las personas que no presentan depresión tienen menor puntaje que aquellas que presentan algún tipo de depresión medido por el BDI-II (Da Silva, Ramos, \& Carvalho, 2018; Leyhe et al., 2017).

Mientras los datos previos no referían alteraciones en la atención y memoria, en este estudio se verifica además de estas, alteraciones en el lenguaje, considerando que la orientación y la atención son mecanismos cognitivos interdependientes (Leyhe et al., 2017). Es igualmente importante referir que los datos previos fueron recogidos en países europeos, los que por cuestiones socioculturales podrían arrojar algunas diferencias asociadas al factor cultural (Riddle et al., 2017).

En lo que respecta a la personalidad se pudo verificar que, cuando existe depresión de tipo moderada, se verifica una diferencia estadísticamente significativa en el rasgo de responsabilidad. Este rasgo se relaciona con la capacidad de planificar el futuro, siendo que su alteración provoca limitaciones en la capacidad que el mayor pueda presentar para el cambio de enfoque de la temática conforme desee y en la capacidad de suprimir respuestas inadecuadas. Además, se puede indagar que los mayores con este tipo de alteración presentan menor longevidad, por su menor motivación para involucrarse en comportamientos que promuevan la salud (Costa, Terracciano, \& McCrae, 2001; Costa \& Widiger, 2002).

Este rasgo se ve aún más afectado, debido a las alteraciones cognitivas, fundamentalmente con los cambios en la atención que evaden la capacidad de seleccionar los estímulos más importantes del entorno; en el lenguaje que por si limita la comunicación del adulto mayor con los cuidadores y profesionales de salud; y la memoria, alejándole de su patrón normal de comportamientos, de sus motivaciones, de sus intereses, y principalmente, de su preocupación por si, por los otros y por su entorno. Todas estas alteraciones se amplifican por la influencia de la depresión, que afecta a los rasgos de personalidad, bien como a los procesos cognitivos (Da Silva, Ramos, \& Carvalho, 2018; Costa \& Widiger, 2002; Leyhe et al., 2017).

En este estudio fue posible verificar un cambio significativo lo que respecta a rasgos de personalidad en el adulto mayor con TNC leve, aunque no se pudo sistematizar un patrón de cambios. El rasgo Responsabilidad disminuye significativamente, juntamente con el DCL del adulto mayor. Conforme indican los resultados, serían los procesos cognitivos los responsables de los cambios comportamentales, considerando que la disminución de valores en el Rasgo Responsabilidad podría estar asociada a cambios en procesos como: orientación, atención y calculo, lenguaje, capacidades viso-constructivas y memoria (Da Silva, Ramos, \& Carvalho, 2018; Leyhe et al., 2017).

Los resultados obtenidos ponen en evidencia una serie de alteraciones funcionales de entidad en el adulto mayor, como por ejemplo la incapacidad de planificar su futuro, de elaborar cambios de enfoque temático en una conversación o en la supresión de respuestas inadecuadas. Aunque, lo más importante de esta investigación es verificar que la depresión afecta tanto a la personalidad como a la cognición, en ese sentido, para un buen diagnóstico diferencial del adulto mayor, es indispensable Intervenir sobre la depresión o considerar sus efectos a la hora de elaborar un diagnóstico y un pronóstico y atender a que, esas variables pueden tener una influencia directa en sus limitaciones comportamentales. (Leyhe et al., 2017; Riddle et al., 2017). 


\section{Referencias}

Beck, A. T., Steer, R. A., \& Brown, G. K. (1996). BDI-II. Beck Depression Inventory- Second Edition. Manual. San Antonio, TX: The Psychological Corporation.

Beck, A. T., Ward, C. H., Mendelson, M., Mock, J., \& Erbaugh, J. (1961). An inventory for measuring depression. Archives of General Psychiatry, 4, 561-571. doi:10.1001/ archpsyc.1961.01710120031004

Cloninger C. R., Przybeck T. R., Svrakic D. M., \& Wetzel R. D. (1994). The Temperament and Character Inventory (tci): A Guide to its Development and Use. Washington: Center for Psychology of Personality.

Costa, P. T., Terracciano, A., \& McCrae, R. R. (2001). Gender diferences in personality traits across cultures. Robust and surprising fndings. Journal of Personality and Social Psychology, 81(2), 322-331. doi: 10.1037/00223514.81.2.322.

Costa, P. T., \& Widiger, T. A. (Eds.). (2002). Personality disorders and the fve-factor model of personality (2nd ed.). Washington, DC: American Psychological Association.

Da Silva, C. Y. R. (2017). Envejecimiento - Evaluación e Intervención. México: Manual Moderno.

Da Silva, C. Y. \& Carvalho, P. A. (2017a). Influencia de la depresión en cambios de personalidad del adulto mayor con enfermedad de Alzheimer incipiente. Enseñanza e Investigación en Psicología, 22(1), 103-109.

Da Silva, C. Y. \& Carvalho, P. A. (2017b). Estado conductual y psiquiátrico en el adulto mayor con trastorno neurocognitivo leve de enfermedad de Alzheimer posible. Enseñanza e Investigación en Psicología, 22(3), 373-379.

Da Silva, C. Y., Carvalho, P. A., González, S. L. L., \& Quino, H. J. M. (2018). Personalidad y estado psicológico en el trastorno neurocognitivo leve debido a enfermedad de Alzheimer posible. Revista Alternativas en Psicología, 39(1): $104-115$.

Da Silva, C. Y., Carvalho, P. A., Quino, H. J. M., \& Gruart, M. V. (2017). Effects of depression on cognition of patients with mild neurocognitive disorder due to possible Alzheimer's disease. Ciencias psicológicas, 11(2): 149 - 154. doi: 10.22235/cp. v11i2.1486.

Da Silva, C. Y., Ramos, V. F., \& Carvalho, P. A. (2018). Incremento de los cambios cognitivos en pacientes con enfermedad de Alzheimer inicial debido a depresión. Panamerican Journal of Neuropsychology, 12(1): 42 - 51. doi: 10.7714/CNPS/12.1.203.

Folstein, M. F., Folstein, S. E., \& McHugh, P. R. (1975). Mini-Mental State: a practical method for grading the cognitive state of patients for clinician. Journal Psychiatric Reserch, 12: 189-198. doi.org/10.1016/00223956(75)90026-6.

Leyhe, T., Reynolds, C. F., Melcher, T., Linnemann, C, Klöppel, S., Blennow, K., Zetterberg, H. ... Hampel, H. (2017). A common challenge in older adults: Classification, overlap, and therapy of depression and dementia. The Journal of the Alzheimer's Association, 13(1): 59-71. doi.org/10.1016/j.jalz.2016.08.007.
Pérez-Fuentes, M. C., Gázquez, J. J., \& Morelo, M. M. (2012). Análisis de los "cinco grandes" factores de la personalidad en alumnos de la Universidad de mayores de Almería. European Journal of Investigation in Health Psychology and Education, 2(1), 19-28.

Pocnet, C., Rossier, J., Antonietti, J. P., Gunten A., \& Phill, M. (2011). Personality Changes in Patients with Beginning Alzheimer Disease. La Revue canadienne de psychiatrie, 56(7): 408-417.

Qazi, A., Gutzmann, H., \& Gul S. (2017) Depression and Anxiety in Dementia Subjects. In: Verdelho A., GonçalvesPereira M. (eds,) Neuropsychiatric Symptoms of Cognitive Impairment and Dementia. Neuropsychiatric Symptoms of Neurological Disease. Springer, Cham. doi: 10.1007/s00391-015-0898-8.

Ramakers, I. H. G. B., \& Verhey F. R. J. (2017) The Difficult Distinction Between Affective Disorders and Mild Cognitive Deterioration. In: Verdelho A., Gonçalves-Pereira M. (eds.) Neuropsychiatric Symptoms of Cognitive Impairment and Dementia. Neuropsychiatric Symptoms of Neurological Disease. Springer, Cham.

Riddle, M., Potter, G. G., McQuoid, D. R., Steffens, D. C., Beyer, J. L., \& Taylor W. D. (2017). Longitudinal Cognitive Outcomes of Clinical Phenotypes of LateLife Depression. The American Journal of Geriatric Psychiatry, 25(10): 1123-1134. doi: 10.1016/j.jagp.

Rodrigues, C. (2015). Personalidad de Alzheimer - conducta y estado psicológico. España: Publicia.

Rodrigues, C., Castro, F. V., \& Cruz Roja Española. (2014). Los cambios de personalidad en la enfermedad de Alzheimer. International Journal of Developmental and Educational Psychology, INFAD, 5(1): 177-186.

Rodrigues, C., Castro, F. V., \& Figueiredo, P. (2014). Aspectos de la personalidad y personalidad premórbida en la enfermedad de Parkinson. International Journal of Developmental and Educational Psychology, INFAD, 5(1): 195-204

Rodrigues, C., Castro, F. V., \& Gruart, M. (2014a). Relación entre Cognición y Personalidad en Enfermos con Alzheimer. International Journal of Developmental and Educational Psychology, INFAD, 1(2): 187-196.

Rodrigues, C., Castro, F. V., \& Gruart, M. (2014b). La Personalidad premórbida en la enfermedad de Alzheimer. International Journal of Developmental and Educational Psychology, INFAD, 1(2): 283-292.

Vidovich, M. \& Almeida, O. P. (2011). Cognition-focused interventions for older adults: the state of play. Australasian Psychiatry 19(4): 313-316. doi: 10.3109/10398562.2011.579973. 